\title{
Admissibility Analysis and Controller Design for Discrete Singular Time-Delay Systems Embracing Uncertainties in the Difference and Systems' Matrices
}

\author{
Chih-Peng Huang (iD \\ Department of Computer Science, University of Taipei, No. 1, Ai-Guo West Rd, Taipei City 100, Taiwan \\ Correspondence should be addressed to Chih-Peng Huang; cphuang@utaipei.edu.tw
}

Received 10 September 2020; Revised 15 December 2020; Accepted 22 December 2020; Published 20 January 2021

Academic Editor: Luigi Rodino

Copyright ( 2021 Chih-Peng Huang. This is an open access article distributed under the Creative Commons Attribution License, which permits unrestricted use, distribution, and reproduction in any medium, provided the original work is properly cited.

\begin{abstract}
This paper mainly investigates the admissibility analysis and the admissibilizing controller design for the uncertain discrete singular system with delayed state. Based on Lyapunov-Krasovskii stability theory, an original admissibility condition for the nominal singular delay system is first presented. By involving the uncertainties in both difference and system matrices simultaneously, we devote to analyzing the robust admissibility for the regarded uncertain discrete singular system with delayed state. Furthermore, by hiring the state feedback control law, we further discuss the admissibilizing controller design for the resulting closed-loop system. Since all the derived criteria are expressed in terms of strict linear matrix inequalities (LMIs) or parametric LMIs, we thus can handily verify them via current LMI solvers. Finally, two numerical examples are given to illustrate the effectiveness and validity of the proposed approach.
\end{abstract}

\section{Introduction}

During recent years, the singular systems have received extensive attention because they can represent a wider class of physical systems, including physical models and nondynamic constraints, than traditional state-space systems [1]. They can represent large-scale systems [2], economic systems [3, 4], electrical network analysis [5], and so on. Singular systems are also referred to as descriptor systems, implicit systems, generalized state-space systems, differential-algebraic systems, or semistate systems $[2,6-8]$. Recently, robust admissibility and robust controller synthesis for uncertain singular systems have been addressed. The corresponding stability issues are more complicated than the traditional state-space system because they require considering not only the stability but also the regularity and causality simultaneously (see, for example, [9-18] and the references therein).

Furthermore, time-delayed state often inevitably exists in many practical systems, such as remote-controlled robots, chemical processes, and electrical network grids. They are the main sources of instability, oscillations, or degraded performances [19-23]. Therefore, the problems of stability testing and stabilization of uncertain singular systems with delay states have aroused much attention over the recent years, e.g., [24-36]. For discrete-time systems, Xu et al. [24] proposed specific D-stability issues for uncertain discrete singular systems with state delay. Feng et al. [30] discussed the admissibility for discrete singular systems with timevarying delay and attained the admissibility criterion with strict linear matrix inequalities (LMIs). Also, the robust admissibility and admissibilization of uncertain discrete singular time-delay systems were further investigated [31]. In [32], novel delay-dependent sufficient conditions for the robust finite-time stability of the system are presented. Then, the results can be applied to solve the finite-time stabilization problem of linear singular discrete-time control delay systems. In [33], based on the equivalent restrict decomposition form, the iterative learning control problem is studied for a class of discrete singular time-delay systems. Also, by the LMI approach, an efficient observer design method is presented for singular discrete-time systems with time delays 
and nonlinearity [34]. In [36], this work proposes a novel LMI-based global sliding mode control approach for uncertain discrete-time descriptor systems with multiple timevarying delays. However, in previous works, they all focus on the uncertain singular systems with a constant difference matrix or the difference matrix satisfying a prescribed form. For modeling singular systems from physical systems, if uncertainties exist in a structure or a behavior, they are inevitably cast into the systems' matrices and the derivative matrix (continuous systems) or the difference matrix (discrete systems) simultaneously [37-40]. To the best of our knowledge, the discrete singular delay systems with the uncertainties existing in both difference matrix $E$ and the system matrices seem little to be discussed in the past literature studies.

In this work, we devote to analyzing the robust admissibility and the admissibilizing controller design for the discrete delayed singular system with the perturbed difference and system matrices simultaneously. Based on the Lyapunov stability theory, an admissibility condition is first derived for the nominal singular system with delayed state. Thus, the robust admissibility criteria can be attained for the system with the allowed uncertainties in both difference and systems' matrices. Furthermore, when hiring the state feedback control law, we further address controller design criteria for the resulting closed-loop systems. For numerical evaluation, all proposed criteria are represented by strict or parametric LMIs [41], and we can readily verify them via a current LMI solver [42]. At length, two numerical examples are given to illustrate the validity and practicability of the proposed approach. Compared with previous works, the main contributions of this paper are highlighted as follows:

(i) In the past, it seems that there are few results on analyzing the robust admissibility and the admissibilizing controller design for the discrete delayed singular system with embracing the uncertainties in both difference and system matrices simultaneously. This work mainly copes with the admissibility analysis and admissibilizing controller design for the regarded system with the perturbed derivative matrix.

(ii) All the proposed admissibility analyses and controller design criteria are all explicitly formulated in terms of LMIs or parametric LMIs, so we can readily verify them via LMI solvers for the admissibility assurance or systematically performing a controller design. The validity and efficiency of the proposed approach are demonstrated by illustrative examples.

\section{Problem Formulation and Preliminaries}

Consider a discrete uncertain singular delay system described by

$$
\left\{\begin{array}{l}
\widetilde{E} x(k+1)=(A+\Delta A) x(k)+\left(A_{d}+\Delta A_{d}\right) x(k-d)+(B+\Delta B) u(k), \quad k>0, \\
x(i)=\phi(i), \quad i=-d,-d+1, \ldots, 0,
\end{array}\right.
$$

where $x(\cdot) \in R^{n}$ is the state vector and $u(\cdot) \in R^{p}$ is the control input; the perturbed derivative matrix $\widetilde{E} \in R^{n \times n}$ may be singular, i.e., $\operatorname{rank}(\widetilde{E})=m \leq n$, and belongs to a polytopic set defined as

$$
\Omega_{E} \triangleq\left\{\widetilde{E}: \widetilde{E}=\sum_{i}^{r} e_{i} E_{i}, e_{i} \geq 0, \sum_{i=1}^{r} e_{i}=1\right\},
$$

where $E_{i} \in R^{n \times n}$ given a priori. $A, A_{d}$, and $B$ stand for the nominal system matrices with appropriate dimensions, and $\Delta A, \Delta A_{d}$, and $\Delta B$ represent the parameter uncertainties bounded by

$$
\left[\begin{array}{lll}
\Delta A^{T} & \Delta A_{d}^{T} & \Delta B
\end{array}\right]=\operatorname{MD}\left[\begin{array}{lll}
N_{A} & N_{\text {Ad }} & N_{B}
\end{array}\right],
$$

where $M, N_{A}, N_{A d}$, and $N_{B}$ are constant matrices with compatible dimensions and $D$ satisfies $D^{T} D \leq I$

Remark 1 (see [40]). For system's model (1), the presented uncertainties in the derivative matrix and the system matrices are formulated by different forms. For mathematical modeling from a physical system, if there exists parametric perturbation within its inner structures or behaviors, the main parametric uncertainties can usually be cast into system matrices, and the remaining few uncertainties are thus cast into the difference matrix. So, we can reasonably formulate the system's matrices with whole uncertainties by the norm bound in equation (3) and the difference matrix with individual uncertainties by the polytopic form in equation (2). A circuit example can be found in Example 1 in the previous work [40].

To investigate the stability and design issues for system (1), definitions for the discrete singular system are given as follows.

Definition 1 (see $[8,24])$.

(I) The pair $(E, A)$ is said to be $\operatorname{regular}$ if $\operatorname{det}(z E-A)$ is not identically zero

(II) The pair $(E, A)$ is said to be causal if it is regular and $\operatorname{deg}(\operatorname{det}(z E-A))=\operatorname{rank}(E)$

(III) Let $\rho(E, A) \triangleq \max _{\lambda \in\{z \mid \operatorname{det}(z E-A)=0\}}|\lambda|$; the pair $(E, A)$ is said to be stable if $\rho(E, A)<1$

(IV) The pair $(E, A)$ is said to be admissible if it is regular, causal, and stable 
Definition 2 (see $[8,24]$ ).

(I) The triple $\left(E, A, A_{d}\right)$ is said to be regular if $\operatorname{det}\left(z^{d+1} E-z^{d} A-A_{d}\right)$ is not identically zero

(II) The triple $\left(E, A, A_{d}\right)$ is said to be causal if it is regular and $\operatorname{deg}\left(z^{n d} \operatorname{det}\left(z E-A-z^{-d} A_{d}\right)\right)$ $=n d+\operatorname{rank}(E)$

(III) The discrete singular delay system $E x(k+1)=A x(k)+A_{d} x(k-d)$ is said to be stable if $\quad \rho\left(E, A, A_{d}\right)<1$, where $\rho\left(E, A, A_{d}\right) \triangleq \max _{\lambda \in\left\{z \mid \operatorname{det}\left(z^{d+1} E-z^{d} A-A_{d}\right)=0\right\}}|\lambda|$

(IV) The discrete singular delay system $E x(k+1)=A x(k)+A_{d} x(k-d)$ (i.e., the triple $\left.\left(E, A, A_{d}\right)\right)$ is said to be admissible if it is regular, causal, and stable

Lemma 1 (see $[8,24])$. ie nominal discrete singular delay system $\operatorname{Ex}(k+1)=A x(k)+A_{d} x(k-d)$ is regular, causal, and stable if and only if the pair $(E, A)$ is regular, causal, and $\rho\left(E, A, A_{d}\right)<1$.

Lemma 2 (see [29]). Ie triple $\left(E, A, A_{d}\right)$ is admissible if and only if the corresponding transpose form $\left(E^{T}, A^{T}, A_{d}^{T}\right)$ is admissible.

\section{Admissibility Analysis and Admissibilizing Controller Design}

3.1. Admissibility Analysis. For analyzing the robust admissibility for system (1), a sufficient condition for the unforced nominal singular system $E x(k+1)=A x(k)+A_{d} x(k-d)$, that is, $\Delta A=\Delta A_{d}=0$ and $u(k) \equiv 0$ in (1) and $\widetilde{E}=E$ in (2), is first derived as follows.
Lemma 3. Unforced nominal discrete singular delay system (1) is admissible if there exist matrices $P>0, R>0$, and $Q$ with appropriate dimensions such that

$$
\left[\begin{array}{ccc}
Q S^{T} A+A^{T} S Q^{T}-E^{T} P E+R & Q^{T} A_{d} & A^{T} P \\
A_{d}^{T} S Q^{T} & -R & A_{d}^{T} P \\
P A & P A_{d} & -P
\end{array}\right]<0,
$$

where $S \in R^{n \times(n-m)}$ is any matrix with full column rank and satisfies $E^{T} S=0$.

Proof. Assume that matrices $P>0, R>0$, and $Q$ satisfy (4). By the Schur complement [41], equation (4) leads to

$$
\left[\begin{array}{cc}
A^{T} P A-E^{T} P E+A^{T} S Q^{T}+Q S^{T} A+R & \left(P A+S Q^{T}\right)^{T} A_{d} \\
A_{d}^{T}\left(P A+S Q^{T}\right) & A_{d}^{T} P A_{d}-R
\end{array}\right]<0 .
$$

For matrix $E$ with $\operatorname{rank}(E)=r \leq n$, there exist nonsingular matrices $M_{1}, N_{1} \in R^{n \times n}$ such that

$$
\begin{aligned}
M_{1} E N_{1} & =\left[\begin{array}{ll}
I_{r} & 0 \\
0 & 0
\end{array}\right], \\
M_{1} A N_{1} & =\left[\begin{array}{ll}
A_{1} & A_{2} \\
A_{3} & A_{4}
\end{array}\right], \\
M_{1}^{-T} P M_{1}^{-1} & =\left[\begin{array}{ll}
P_{1} & P_{2} \\
P_{2}^{T} & P_{3}
\end{array}\right], \\
M_{1}^{-T} S Q^{T} N_{1} & =\left[\begin{array}{ll}
0 & 0 \\
S_{1} & S_{2}
\end{array}\right], \\
N_{1}^{T} R N_{1} & =\left[\begin{array}{ll}
R_{1} & R_{2} \\
R_{2}^{T} & R_{3}
\end{array}\right] .
\end{aligned}
$$

Using the expression in (6a), (6b) and deducing from the block $(1,1)$ in $(5)$, we have

$$
\begin{aligned}
& A^{T} P A-E^{T} P E+A^{T} S Q^{T}+Q S^{T} A+R \\
& =N_{1}^{-T}\left(\left[\begin{array}{ll}
A_{1}^{T} & A_{3}^{T} \\
A_{2}^{T} & A_{4}^{T}
\end{array}\right]\left[\begin{array}{ll}
P_{1} & P_{2} \\
P_{2}^{T} & P_{3}
\end{array}\right]\left[\begin{array}{ll}
A_{1} & A_{2} \\
A_{3} & A_{4}
\end{array}\right]-\left[\begin{array}{ll}
I_{r} & 0 \\
0 & 0
\end{array}\right]\left[\begin{array}{ll}
P_{1} & P_{2} \\
P_{2}^{T} & P_{3}
\end{array}\right]\left[\begin{array}{ll}
I_{r} & 0 \\
0 & 0
\end{array}\right]+\left[\begin{array}{ll}
A_{1}^{T} & A_{3}^{T} \\
A_{2}^{T} & A_{4}^{T}
\end{array}\right]\left[\begin{array}{ll}
0 & 0 \\
S_{1} & S_{2}
\end{array}\right]+\left[\begin{array}{ll}
0 & S_{1}^{T} \\
0 & S_{2}^{T}
\end{array}\right]\left[\begin{array}{ll}
A_{1} & A_{2} \\
A_{3} & A_{4}
\end{array}\right]+\left[\begin{array}{ll}
R_{1} & R_{2} \\
R_{2}^{T} & R_{3}
\end{array}\right]\right) N_{1}^{-1} \\
& =N_{1}^{-T}\left\{\left[\begin{array}{cc}
* & * \\
* & A_{2}^{T} P_{1} A_{2}+A_{4}^{T} P_{2}^{T} A_{2}+A_{2}^{T} P_{2} A_{4}+A_{4}^{T} P_{3} A_{4}+A_{4}^{T} S_{2}+S_{2}^{T} A_{4}+R_{3}
\end{array}\right]\right\} N_{1}^{-1} \\
& =N_{1}^{-T}\left\{\left[\begin{array}{cc}
* & * \\
* & A_{4}^{T}\left(P_{2}^{T} A_{2}+S_{2}\right)+\left(P_{2}^{T} A_{2}+S_{2}\right)^{T} A_{4}+\left(A_{2}^{T} P_{1} A_{2}+A_{4}^{T} P_{3} A_{4}+R_{3}\right)
\end{array}\right]\right\} N_{1}^{-1}<0,
\end{aligned}
$$

where "*" denotes the not utilized matrix. Due to $P_{1}>0$, $P_{3}>0$, and $R_{3}>0$, the least equation implies $A_{4}^{T}\left(P_{2}^{T} A_{2}+S_{2}\right)+\left(P_{2}^{T} A_{2}+S_{2}\right)^{T} A_{4}<0$, i.e., the nonsingularity of $\left(P_{2}^{T} A_{2}+S_{2}\right)^{T} A_{4}$, and so does $A_{4}$. From Definition 1 associated with Lemma 1 , the nominal discrete singular delay system is asserted to be regular and causal.
Choose a Lyapunov-Krasovskii functional candidate for the regarded system as

$$
V(k)=x^{T}(k) E^{T} P E x(k)+\sum_{j=1}^{d} x(k-j)^{T} R x(k-j) .
$$


By the Lyapunov quadratic stability theory, differentiating $V(k)$ can be manipulated as

$$
\begin{aligned}
\Delta V(k)= & V(k+1)-V(k) \\
= & x^{T}(k+1) E^{T} P E x(k+1)+\sum_{j=1}^{d} x(k+1-j)^{T} \\
& R x(k+1-j) \\
& -x^{T}(k) E^{T} P E x(k)-\sum_{j=1}^{d} x(k-j)^{T} R x(k-j) \\
= & x^{T}(k+1) E^{T} P E x(k+1)-x^{T}(k) E^{T} P \operatorname{Ex}(k) \\
& +x^{T}(k) R x(k)-x^{T}(k-d) R x(k-d) .
\end{aligned}
$$

Deducing from $\Delta V(k)$ along the considered system and using the property $E^{T} S=0$ lead to

$$
\begin{aligned}
\Delta V & (k)=x^{T}(k+1) E^{T} P E x(k+1)-x^{T}(k) E^{T} P E x(k) \\
& +x^{T}(k) R x(k)-x^{T}(k-d) R x(k-d) \\
& +x^{T}(k+1) E^{T} S Q^{T} x(k)+x^{T}(k) Q S^{T} E x(k+1) \\
= & \left(A x(k)+A_{d} x(k-d)\right)^{T} P(A x(k) \\
& \left.+A_{d} x(k-d)\right)-x^{T}(k) E^{T} P E x(k) \\
& +x^{T}(k) R x(k)-x^{T}(k-d) R x(k-d) \\
& +\left(A x(k)+A_{d} x(k-d)\right)^{T} S Q^{T} x(k) \\
& +x^{T}(k) Q S^{T}\left(A x(k)+A_{d} x(k-d)\right) \\
= & x^{T}(k)\left(A^{T} P A-E^{T} P E+A^{T} S Q^{T}+Q S^{T} A+R\right) x(k) \\
& +2 x^{T}(k)\left(A^{T} P+Q S^{T}\right) A_{d} x(k-d) \\
& +x^{T}(k-d)\left(A_{d}^{T} P A_{d}-R\right) x(k-d) \\
= & \zeta^{T}(k)\left[\begin{array}{c}
A^{T} P A-E^{T} P E+A^{T} S Q^{T}+Q S^{T} A+R\left(P A+S Q^{T}\right)^{T} A_{d} \\
\quad A_{d}^{T} P A_{d}-R
\end{array}\right] \zeta(k),
\end{aligned}
$$

where $\zeta(k) \triangleq\left[\begin{array}{ll}x^{T}(k) & x^{T}(k-d)\end{array}\right]^{T}$. By the Schur complement, equation (4) is equivalent to

$$
\left[\begin{array}{cc}
A^{T} P A-E^{T} P E+A^{T} S Q^{T}+Q S^{T} A+R & \left(P A+S Q^{T}\right)^{T} A_{d} \\
A_{d}^{T}\left(P A+S Q^{T}\right) & A_{d}^{T} P A_{d}-R
\end{array}\right]<0
$$

and leads to $\Delta V(k)<0$, and the considered system is stable. Associated with regularity and causality, the system is thus concluded to be admissible according to Definition 2 associated with Lemma 1.

Based on Lemma 2, the stability criteria in Lemma 3 for unforced nominal discrete singular delay system (1) can be transformed into an alternative matrices' transpose form from (5) with replacing $E, A$, and $A_{d}$ with $E^{T}, A^{T}$, and $A_{d}{ }^{T}$, respectively, and are presented as follows.

Corollary 1. Unforced nominal discrete singular delay system (1) is admissible if there exist matrices $P>0, R>0$, and $Q$ with appropriate dimensions such that

$$
\left[\begin{array}{ccc}
\mathrm{QS}^{T} A^{T}+A S Q^{T}-E P E^{T}+R & \mathrm{QS}^{T} A_{d}^{T} & A P \\
A_{d} S Q^{T} & -R & A_{d} P \\
P A^{T} & P A_{d}^{T} & -P
\end{array}\right]<0,
$$

where $S \in R^{n \times(n-m)}$ is any matrix with full column rank and satisfies $E S=0$.

Remark 2. The proposed result in Lemma 3 for the nominal discrete delayed system is directly deduced from matrix algebraic manipulation associated with the Lyapunov-Krasovskii stability theory, where based on this form, we can further investigate the regarded systems in (1) embracing uncertainties in both difference and systems' matrices. However, a distinct result in [8] for the nominal discrete delayed system can also be attained by using the argument system approach.

A robust admissibility criterion for unforced uncertain discrete singular system (1) is mainly presented as follows.

Theorem 1. Unforced uncertain discrete singular delay system (1) is admissible if there exist matrices $P>0, R>0$, and $Q$ with appropriate dimensions and a scalar $\alpha>0$ such that

$$
\left[\begin{array}{cccc}
2 Q S^{T} A^{T}+2 A S Q^{T}-E_{i} P E_{j}^{T}-E_{j} P E_{i}^{T}+2 R+\alpha N_{A}^{T} N_{A} & 2 Q S^{T} A_{d}^{T}+\alpha N_{A}^{T} N_{A d} & A P & 2 Q S^{T} M \\
2 A_{d} S Q^{T}+\alpha N_{A d}^{T} N_{A} & -2 R+\alpha N_{A d}^{T} N_{A d} & A_{d} P & 0 \\
P A^{T} & P A_{d}^{T} & -\frac{1}{2} P & P M \\
2 M^{T} S Q^{T} & & M^{T} P & -\alpha I
\end{array}\right]<0, \quad \forall i \leq j,
$$


where $S \in R^{n \times(n-m)}$ is any matrix with full column rank and satisfies $E_{i} S=0 \forall i$.
Proof. Suppose that there exist matrices $P>0, R>0$, and $Q$ such that (13) holds. By (13) associated with the uncertain matrix $\widetilde{E}$ with $e_{i} \geq 0 \forall i, \sum_{i} e_{i}=1$ defined in (2), we have

$$
\begin{aligned}
& {\left[\begin{array}{cccc}
2 Q S^{T} A^{T}+2 A S Q^{T}-2 \widetilde{E} P \widetilde{E}^{T}+2 R+\alpha N_{A}^{T} N_{A} & 2 Q S^{T} A_{d}^{T}+\alpha N_{A}^{T} N_{A d} & A P & 2 Q S^{T} M \\
2 A_{d} S Q^{T}+\alpha N_{A d}^{T} N_{A} & -2 R+\alpha N_{A d}^{T} N_{A d} & A_{d} P & 0 \\
P A^{T} & P A_{d}^{T} & -\frac{1}{2} P & P M \\
2 M^{T} S Q^{T} & 0 & M^{T} P & -\alpha I
\end{array}\right]}
\end{aligned}
$$

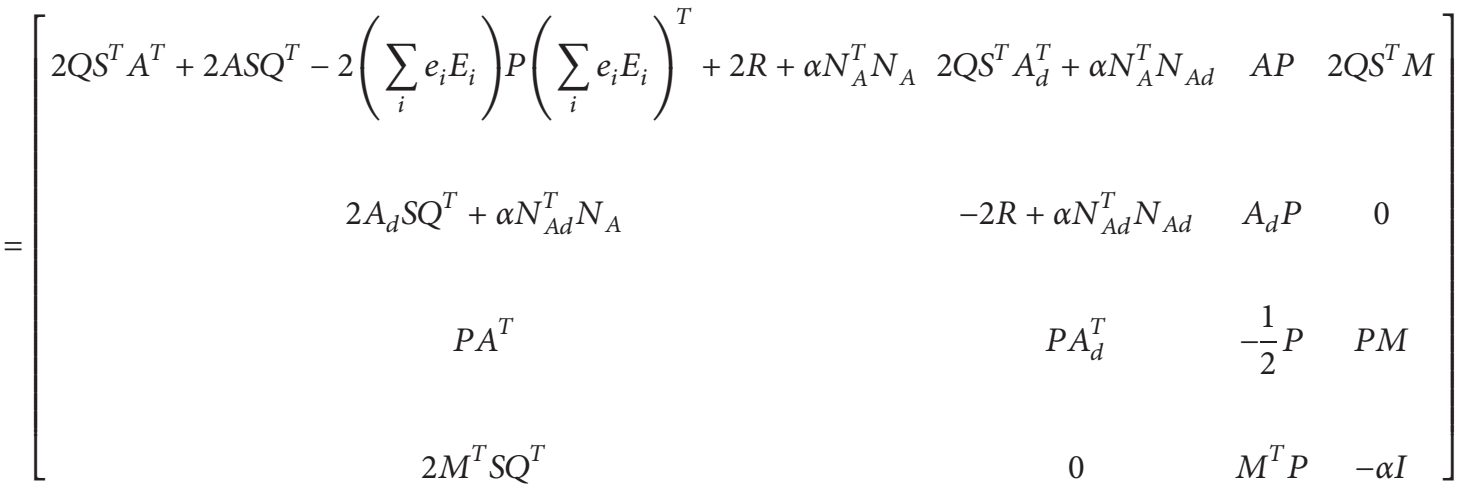

$$
\begin{aligned}
& =\sum_{i} e_{i}^{2}\left[\begin{array}{cccc}
2 Q S^{T} A^{T}+2 A S Q^{T}-2 E_{i} P E_{i}^{T}+2 R+\alpha N_{A}^{T} N_{A} & 2 Q S^{T} A_{d}^{T}+\alpha N_{A}^{T} N_{A d} & A P & 2 Q S^{T} M \\
2 A_{d} S Q^{T}+\alpha N_{A d}^{T} N_{A} & -2 R+\alpha N_{A d}^{T} N_{A d} & A_{d} P & 0 \\
P A^{T} & P A_{d}^{T} & -\frac{1}{2} P & P M \\
2 M^{T} S Q^{T} & & M^{T} P & -\alpha I
\end{array}\right]
\end{aligned}
$$

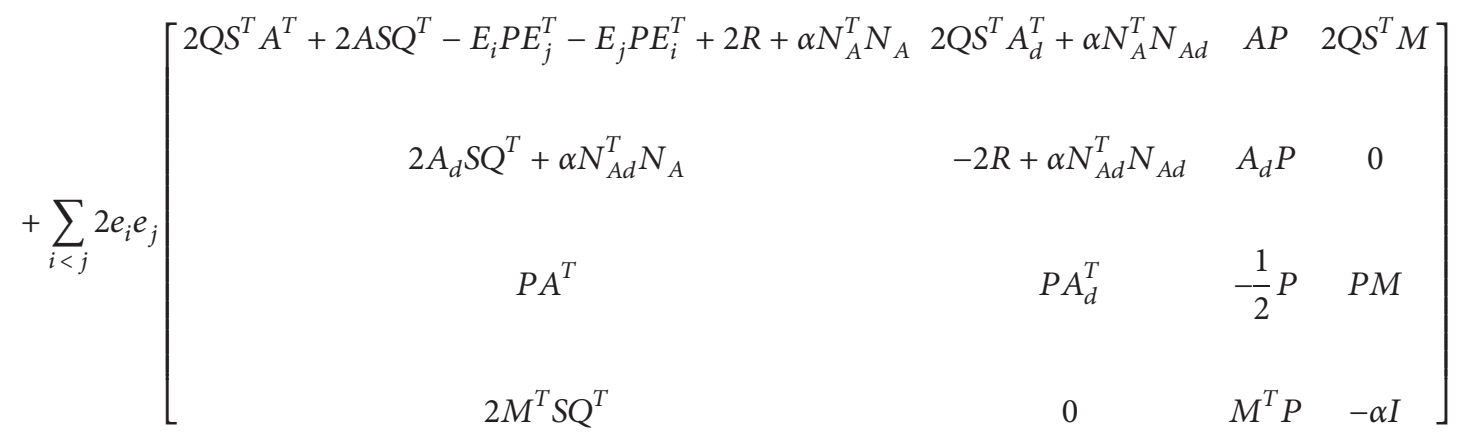


Deducing from Corollary 1 associated with the uncertain matrices $\Delta A$ and $\Delta A_{d}$ defined in (3), we have

$$
\begin{aligned}
& {\left[\begin{array}{ccc}
2 Q S^{T}(A+\Delta A)^{T}+2(A+\Delta A) S Q^{T}-2 \widetilde{E} P \widetilde{E}^{T}+2 R & 2 Q S^{T}\left(A_{d}+\Delta A_{d}\right)^{T} & (A+\Delta A) P \\
2\left(A_{d}+\Delta A_{d}\right) S Q^{T} & -2 R & \left(A_{d}+\Delta A_{d}\right) P \\
P(A+\Delta A)^{T} & P\left(A_{d}+\Delta A_{d}\right)^{T} & -\frac{1}{2} P
\end{array}\right]}
\end{aligned}
$$

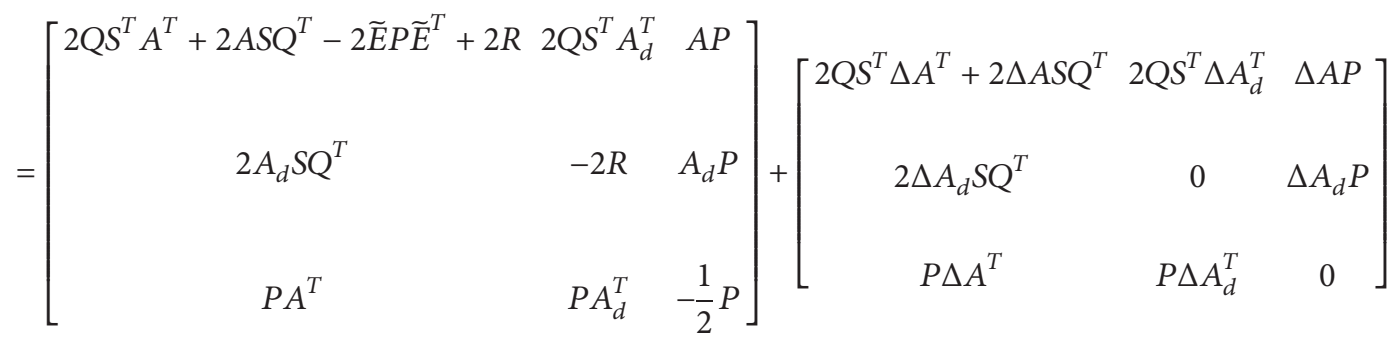

$$
\begin{aligned}
& =\left[\begin{array}{ccc}
2 Q S^{T} A^{T}+2 A S Q^{T}-2 \widetilde{E} P \widetilde{E}^{T}+2 R & 2 Q S^{T} A_{d}^{T} & A P \\
2 A_{d} S Q^{T} & -2 R & A_{d} P \\
& & \\
P A^{T} & P A_{d}^{T} & -\frac{1}{2} P
\end{array}\right] \\
& +\left[\begin{array}{c}
2 Q S^{T} M \\
0 \\
P M
\end{array}\right] D\left[\begin{array}{lll}
N_{A} & N_{A d} & 0
\end{array}\right]+\left[\begin{array}{c}
N_{A}^{T} \\
N_{A d}^{T} \\
0
\end{array}\right] D^{T}\left[\begin{array}{lll}
2 M^{T} S Q & 0 & M^{T} P
\end{array}\right] \\
& \leq\left[\begin{array}{ccc}
2 Q S^{T} A^{T}+2 A S Q^{T}-2 \widetilde{E} P \widetilde{E}^{T}+2 R & 2 Q S^{T} A_{d}^{T} & A P \\
2 A_{d} S Q^{T} & -2 R & A_{d} P \\
& & \\
P A^{T} & P A_{d}^{T} & -\frac{1}{2} P
\end{array}\right] \\
& +\alpha^{-1}\left[\begin{array}{c}
2 Q S^{T} M \\
0 \\
P M
\end{array}\right]\left[\begin{array}{lll}
2 M^{T} S Q & 0 & M^{T} P
\end{array}\right]+\alpha\left[\begin{array}{c}
N_{A}^{T} \\
N_{A d}^{T} \\
0
\end{array}\right]\left[\begin{array}{lll}
N_{A} & N_{A d} & 0
\end{array}\right] .
\end{aligned}
$$


By the Schur complement associated with the aforementioned negative definite inequality, the above is equivalent to

$$
\left[\begin{array}{cccc}
2 Q S^{T} A^{T}+2 A S Q^{T}-2 \widetilde{E} P \widetilde{E}^{T}+2 R+\alpha N_{A}^{T} N_{A} & 2 Q S^{T} A_{d}^{T}+\alpha N_{A}^{T} N_{A d} & A P & 2 Q S^{T} M \\
2 A_{d} S Q^{T}+\alpha N_{A d}^{T} N_{A} & -2 R+\alpha N_{A d}^{T} N_{A d} & A_{d} P & 0 \\
P A^{T} & P A_{d}^{T} & -\frac{1}{2} P & P M \\
2 M^{T} S Q^{T} & 0 & M^{T} P & -\alpha I
\end{array}\right]<0
$$

and then ensures

$$
\left[\begin{array}{ccc}
2 Q S^{T}(A+\Delta A)^{T}+2(A+\Delta A) S Q^{T}-2 \widetilde{E} P \widetilde{E}^{T}+2 R & 2 Q S^{T}\left(A_{d}+\Delta A_{d}\right)^{T} & (A+\Delta A) P \\
2\left(A_{d}+\Delta A_{d}\right) S Q^{T} & -2 R & \left(A_{d}+\Delta A_{d}\right) P \\
P(A+\Delta A)^{T} & P\left(A_{d}+\Delta A_{d}\right)^{T} & -\frac{1}{2} P
\end{array}\right]<0 .
$$

Thus, according to Corollary 1, unforced uncertain discrete singular delay system (1) is asserted to be admissible.

Remark 3. The proposed conditions in Theorem 1 are explicitly expressed by LMIs, and we can handily verify them by the current LMI solver [42]. For evaluating feasible solutions, we need to previously construct a set of LMIs by (13), and the total of LMIs with $n$ numbers of $E_{i}$ 's is $n+C_{2}^{n}$. Thus, the computational time is proportional to the sum of LMIs.

3.2. Admissibilizing Controller Design. Consider uncertain discrete singular delay system (1) associated with a state

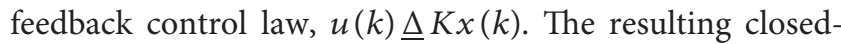
loop system can be formed as

$$
\begin{aligned}
\widetilde{E} x(k+1)= & {[A+\Delta A+(B+\Delta B K)] x(k) } \\
& +\left(A_{d}+\Delta A_{d}\right) x(k-d),
\end{aligned}
$$

where the uncertainties' terms are given in (2) and (3).

In the sequel, we focus on determining a gain matrix $K$ to admissibilize the resulting closed-loop system (18).

Theorem 2. The resulting closed-loop discrete singular delay system (18) is admissible if there exist matrices $P>0, R>0$, and $Q$ with appropriate dimensions and scalars $\alpha>0, \beta>0$, and $a_{l}$ such that

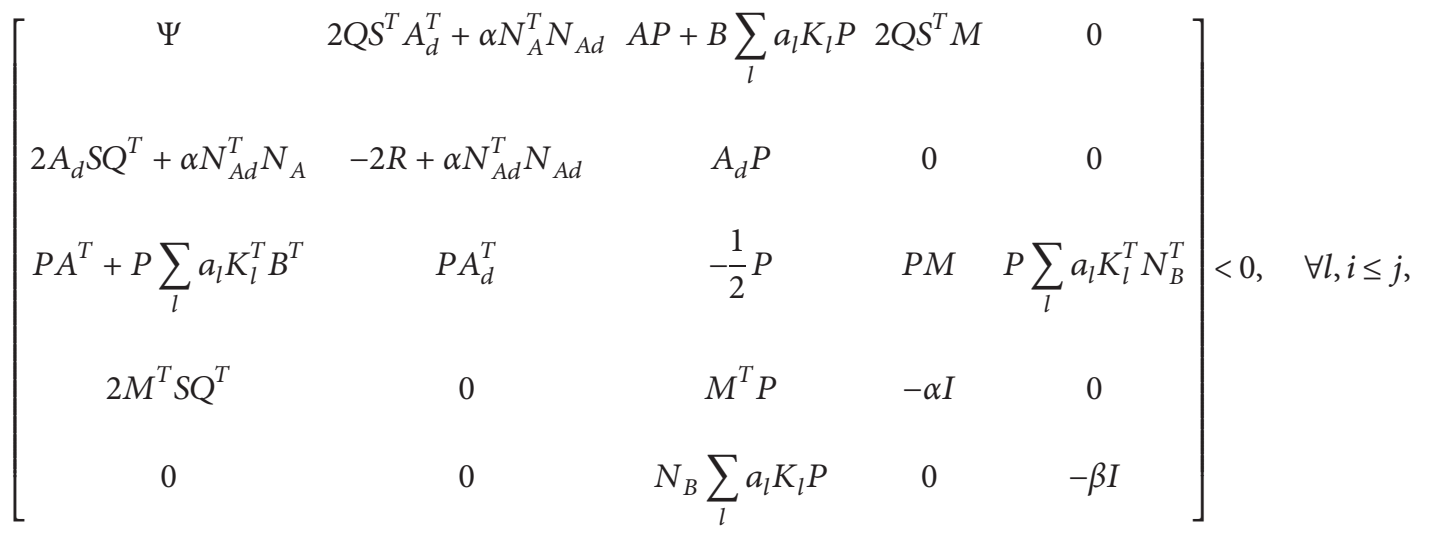


where $\Psi \triangleq 2 Q S^{T} A^{T}+2 A S Q^{T}-E_{i} P E_{j}^{T}-E_{j} P E_{i}^{T}+2 R+\alpha N_{A}^{T}$ $N_{A}+\beta M M^{T}$ and $S \in R^{n \times(n-m)}$ is any matrix with full column rank and satisfies $E_{i} S=0, \forall i$, and an admissibilizing gain matrix is denoted as $K \triangleq \sum_{l} a_{l} K_{l}, K_{l} S=0, \forall l$.
Proof. Based on Theorem 1, the admissible criterion for the resulting closed-loop system (18) with $K \triangleq \sum_{l} a_{l} K_{l}, K_{l} S=0$, $\forall l$, can be constructed by

$$
\begin{aligned}
& {\left[\begin{array}{cccc}
2 Q S^{T}[A+(B+\Delta B) K]^{T}+2[A+(B+\Delta B) K] S Q^{T} & & \\
-E_{i} P E_{j}^{T}-E_{j} P E_{i}^{T}+2 R+\alpha N_{A}^{T} N_{A} & 2 Q S^{T} A_{d}^{T}+\alpha N_{A}^{T} N_{A d} & {[A+(B+\Delta B) K] P} & 2 Q S^{T} M \\
2 A_{d} S Q^{T}+\alpha N_{A d}^{T} N_{A} & -2 R+\alpha N_{A d}^{T} N_{A d} & A_{d} P & \\
P[A+(B+\Delta B) K]^{T} & P A_{d}^{T} & -\frac{1}{2} P & P M \\
2 M^{T} S Q^{T} & & M^{T} P & -\alpha I
\end{array}\right]} \\
& =\left[\begin{array}{cccc}
2 Q S^{T} A^{T}+2 A S Q^{T} & 2 Q S^{T} A_{d}^{T}+\alpha N_{A}^{T} N_{A d} & A P+B \sum_{l} a_{l} K_{l} P & 2 Q S^{T} M \\
-E_{i} P E_{j}^{T}-E_{j} P E_{i}^{T}+2 R+\alpha N_{A}^{T} N_{A} & & \\
2 A_{d} S Q^{T}+\alpha N_{A d}^{T} N_{A} & -2 R+\alpha N_{A d}^{T} N_{A d} & A_{d} P & 0 \\
P A^{T}+P \sum_{l} a_{l} K_{l}^{T} B^{T} & P A_{d}^{T} & -\frac{1}{2} P & P M \\
2 M^{T} S Q^{T} & & M^{T} P & -\alpha I
\end{array}\right] \\
& +\left[\begin{array}{cccc}
0 & 0 & \Delta B \sum_{l} a_{l} K_{l} P & 0 \\
0 & 0 & 0 & 0 \\
P \sum_{l} a_{l} K_{l}^{T} \Delta B^{T} & 0 & 0 & 0 \\
0 & 0 & 0 & 0
\end{array}\right] \\
& =\left[\begin{array}{cccc}
2 Q S^{T} A^{T}+2 A S Q^{T} & 2 Q S^{T} A_{d}^{T}+\alpha N_{A}^{T} N_{A d} & A P+B \sum_{l} a_{l} K_{l} P & 2 Q S^{T} M \\
-E_{i} P E_{j}^{T}-E_{j} P E_{i}^{T}+2 R+\alpha N_{A}^{T} N_{A} & & \\
2 A_{d} S Q^{T}+\alpha N_{A d}^{T} N_{A} & -2 R+\alpha N_{A d}^{T} N_{A d} & A_{d} P & 0 \\
P A^{T}+P \sum_{l} a_{l} K_{l}^{T} B^{T} & P A_{d}^{T} & -\frac{1}{2} P & P M \\
2 M^{T} S Q^{T} & & & \\
& 0 & M^{T} P & -\alpha I
\end{array}\right] \\
& +\left[\begin{array}{l}
M \\
0 \\
0 \\
0
\end{array}\right] D\left[\begin{array}{llll}
0 & 0 & N_{B} \sum_{l} a_{l} K_{l} P & 0
\end{array}\right]+\left[\begin{array}{c}
0 \\
0 \\
P \sum_{l} a_{l} K_{l}^{T} N_{B}^{T} \\
0
\end{array}\right] D^{T}\left[\begin{array}{llll}
M^{T} & 0 & 0 & 0
\end{array}\right]
\end{aligned}
$$




$$
\begin{aligned}
& \leq\left[\begin{array}{cccc}
2 Q S^{T} A^{T}+2 A S Q^{T} & 2 Q S^{T} A_{d}^{T}+\alpha N_{A}^{T} N_{A d} & A P+B \sum_{l} a_{l} K_{l} P & 2 Q S^{T} M \\
-E_{i} P E_{j}^{T}-E_{j} P E_{i}^{T}+2 R+\alpha N_{A}^{T} N_{A} & -2 R+\alpha N_{A d}^{T} N_{A d} & A_{d} P & 0 \\
2 A_{d} S Q^{T}+\alpha N_{A d}^{T} N_{A} & P A_{d}^{T} & -\frac{1}{2} P & P M \\
P A^{T}+P \sum_{l} a_{l} K_{l}^{T} B^{T} & 0 & M^{T} P & -\alpha I
\end{array}\right]
\end{aligned}
$$

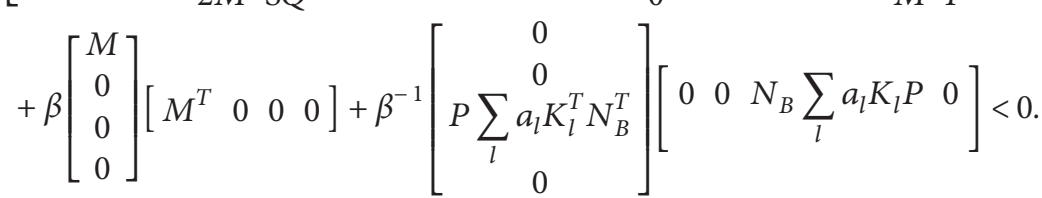

Using the Schur complement in (19), it can directly meet the above. Thus, the resulting closed-loop singular delay system with $K=\sum_{l} a_{l} K_{l}, K_{l} S=0, \forall l$, is asserted to be admissible according to Theorem 1 .

\section{Illustrative Examples}

In this section, we give two illustrative examples to demonstrate the effectiveness and feasibility of the proposed methods.

Example 1. Consider a discrete uncertain singular system with delayed state described by

$$
\left(\left[\begin{array}{ccc}
\varepsilon_{1} & 1 & 0 \\
0 & \varepsilon_{2} & 0 \\
0 & 0 & 0
\end{array}\right]\right) x(k+1)=(A+\Delta A) x(k)+\left(A_{d}+\Delta A_{d}\right) x(k-1),
$$

where $\varepsilon_{1} \in[0.8,1], \quad \varepsilon_{2} \in[1,1.2], \quad A=\left[\begin{array}{ccc}0.4 & 0.2 & 0 \\ 0.3 & 0.3 & 0.4 \\ 0.2 & 0.3 & 0.5\end{array}\right]$, $A_{d}=\left[\begin{array}{ccc}0.2 & 0.1 & 0 \\ 0.1 & 0.3 & 0.3 \\ 0.1 & 0.2 & 0.1\end{array}\right]$, and $\left[\begin{array}{cc}\Delta A^{T} & \Delta A_{d}^{T}\end{array}\right]=\operatorname{MD}\left[\begin{array}{ll}N_{A} & N_{A d}\end{array}\right]$ with $\quad M=\left[\begin{array}{lll}1 & 1 & 1\end{array}\right]^{T}, \quad N_{A}=\left[\begin{array}{lll}0.2 & 0.3 & 0.2\end{array}\right], \quad$ and $N_{A d}=\left[\begin{array}{lll}0.1 & 0.1 & 0.2\end{array}\right]$. Since perturbed difference matrix $\widetilde{E}$ has two uncertain parameters mainly in the diagonal elements, according to (2), we can denote matrices as

$$
E_{1}=\left[\begin{array}{ccc}
0.8 & 1 & 0 \\
0 & 1 & 0 \\
0 & 0 & 0
\end{array}\right]
$$

$$
E_{2}=\left[\begin{array}{lll}
1 & 1 & 0 \\
0 & 1 & 0 \\
0 & 0 & 0
\end{array}\right]
$$$$
E_{3}=\left[\begin{array}{ccc}
0.8 & 1 & 0 \\
0 & 1 & 0 \\
0 & 0 & 0
\end{array}\right]
$$

$$
E_{4}=\left[\begin{array}{ccc}
0.8 & 1 & 0 \\
0 & 1.2 & 0 \\
0 & 0 & 0
\end{array}\right]
$$

Due to the regarded discrete singular uncertain system with delayed state includes the uncertainties in both the difference matrix and the systems' matrices, past results [24-36] with a constant $E$ cannot be applied for admissibility verification. However, by Theorem 1 with the given matrix $S=\left[\begin{array}{lll}0 & 0 & 1\end{array}\right]^{T}$ satisfying $E_{i} S=0, \forall i$, the admissibility analysis criteria for the considered system can be represented as a set of strict LMI conditions by (13). When hiring the LMI tool [42] for solving, a set of feasible solutions can be attained and presented as 


$$
\begin{aligned}
P & =\left[\begin{array}{lll}
42.8072 & -21.1936 & -9.9868 \\
-21.1936 & 30.1259 & -11.4180 \\
-9.9868 & -11.4180 & 26.9956
\end{array}\right]>0, \\
R & =\left[\begin{array}{lll}
9.4131 & 4.1940 & 1.0356 \\
4.1940 & 17.5723 & 2.3538 \\
1.0356 & 2.3538 & 3.8766
\end{array}\right]>0, \\
Q & =\left[\begin{array}{l}
-2.1728 \\
-7.6921 \\
-14.5536
\end{array}\right], \\
\alpha & =83.2608>0 .
\end{aligned}
$$

Thus, the considered system ensures to be admissible for all the allowable uncertainties according to Theorem 1.

Example 2. Consider an uncertain discrete singular system with delayed state and control input represented by

$$
\begin{aligned}
& \left(\left[\begin{array}{ccc}
\varepsilon_{1} & 1 & 0 \\
0 & \varepsilon_{2} & 0 \\
0 & 0 & 0
\end{array}\right]\right) x(k+1)=(A+\Delta A) x(k) \\
& \quad+\left(A_{d}+\Delta A_{d}\right) x(k-1)+(B+\Delta B) u(k),
\end{aligned}
$$

where $\varepsilon_{1} \in[1,1.5], \varepsilon_{2} \in[1,1.1]$,

$$
\begin{aligned}
A & =\left[\begin{array}{ccc}
1.2 & 1 & 0.6 \\
0.2 & 1.3 & 0.2 \\
-0.1 & 0.5 & 0.8
\end{array}\right], \\
A_{d} & =\left[\begin{array}{lll}
0.2 & 0.3 & 0 \\
0.2 & 0.2 & 0.1 \\
-0.2 & 0.1 & 0.2
\end{array}\right], \\
B & =\left[\begin{array}{l}
1 \\
1 \\
0
\end{array}\right],
\end{aligned}
$$

and $\quad\left[\begin{array}{lll}\Delta A^{T} & \Delta A_{d}^{T} & \Delta B\end{array}\right]=\operatorname{MD}\left[\begin{array}{lll}N_{A} & N_{A d} & N_{B}\end{array}\right] \quad$ with $M=\left[\begin{array}{lll}0.2 & 0.2 & 0.1\end{array}\right]^{T} N_{A}=\left[\begin{array}{lll}0.2 & 0.2 & 0.2\end{array}\right], N_{A d}=\left[\begin{array}{lll}0.2 & 0.1 & 0.2\end{array}\right]$, and $\mathrm{N}_{\mathrm{B}}=0.2$.

Since perturbed difference matrix $\widetilde{E}$ has two uncertain parameters, according to (2), we can denote matrices $E_{i}$ as

$$
\begin{aligned}
& E_{1}=\left[\begin{array}{lll}
1 & 1 & 0 \\
0 & 1 & 0 \\
0 & 0 & 0
\end{array}\right], \\
& E_{2}=\left[\begin{array}{lll}
1.5 & 1 & 0 \\
0 & 1 & 0 \\
0 & 0 & 0
\end{array}\right], \\
& E_{3}=\left[\begin{array}{lll}
1 & 1 & 0 \\
0 & 1.1 & 0 \\
0 & 0 & 0
\end{array}\right], \\
& E_{4}=\left[\begin{array}{ccc}
1.5 & 1 & 0 \\
0 & 1.1 & 0 \\
0 & 0 & 0
\end{array}\right] .
\end{aligned}
$$

For the unforced singular delay system, i.e., the considered system with $u(t) \triangleq 0$, the states' variables with a given initial condition $\bar{x}(0)=\left[\begin{array}{lll}5 & -4 & -1.5\end{array}\right]^{T}$ are first simulated and depicted in Figure 1. It shows that the states' trajectories are unstable, and a control law needs to be implemented. Since the regarded system embraces two uncertain parameters in difference matrix $\widetilde{E}$, all the past works [24-36] are not suitable. However, according to the result in Theorem 2 with giving a priori matrix $S=\left[\begin{array}{lll}0 & 0 & 1\end{array}\right]^{T}$ satisfying $E_{i} S=0, \forall i$, and $K_{1}=\left[\begin{array}{lll}1 & 0 & 0\end{array}\right]$, $K_{2}=\left[\begin{array}{lll}0 & 1 & 0\end{array}\right]$, a design procedure with the state feedback control can be started up and performed by a set of parametric LMIs by (19). By hiring the LMI solver with given parametric intervals as $a_{1} \in[-5,5]$ and $a_{2} \in[-5,5]$, we thus attain a set of feasible solutions as

$$
\begin{aligned}
& a_{1}=1.1 \text {, } \\
& a_{2}=-1.9 \text {, } \\
& \alpha=0.7415>0 \text {, } \\
& \beta=0.7392>0 \text {, } \\
& P=\left[\begin{array}{ccc}
30.7692 & 32.5080 & -24.6526 \\
32.5080 & 60.2108 & -25.2156 \\
-24.6526 & -25.2156 & 39.3956
\end{array}\right] \times 10^{-2}>0 \text {, } \\
& R=\left[\begin{array}{ccc}
35.7666 & 22.4145 & 6.1015 \\
22.4145 & 17.2951 & -0.2599 \\
6.1015 & -0.2599 & 19.3578
\end{array}\right] \times 10^{-2}>0 \text {, } \\
& Q=\left[\begin{array}{c}
16.4837 \\
15.3854 \\
-37.8544
\end{array}\right] \times 10^{-2} \text {. }
\end{aligned}
$$

Then, an admissibilizing controller gain can be consequently determined by

$$
K=\sum_{l} a_{l} K_{l}=a_{1} K_{1}+a_{2} K_{2}=\left[\begin{array}{lll}
1.1 & -1.9 & 0
\end{array}\right] .
$$

Thus, when denoting the same initial condition as the above, the considered system which equipped the state 


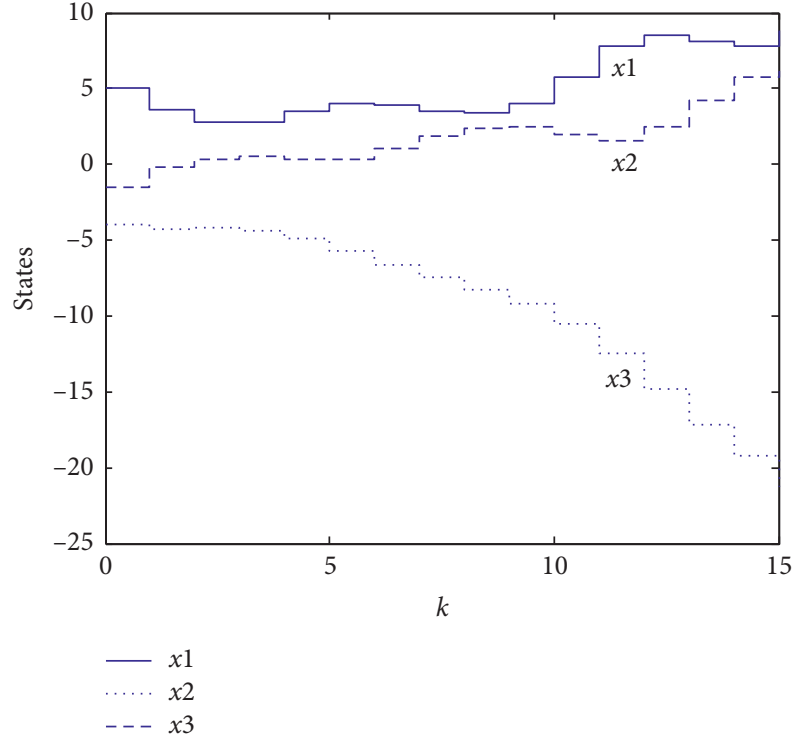

Figure 1: Unforced states' responses for Example 2.

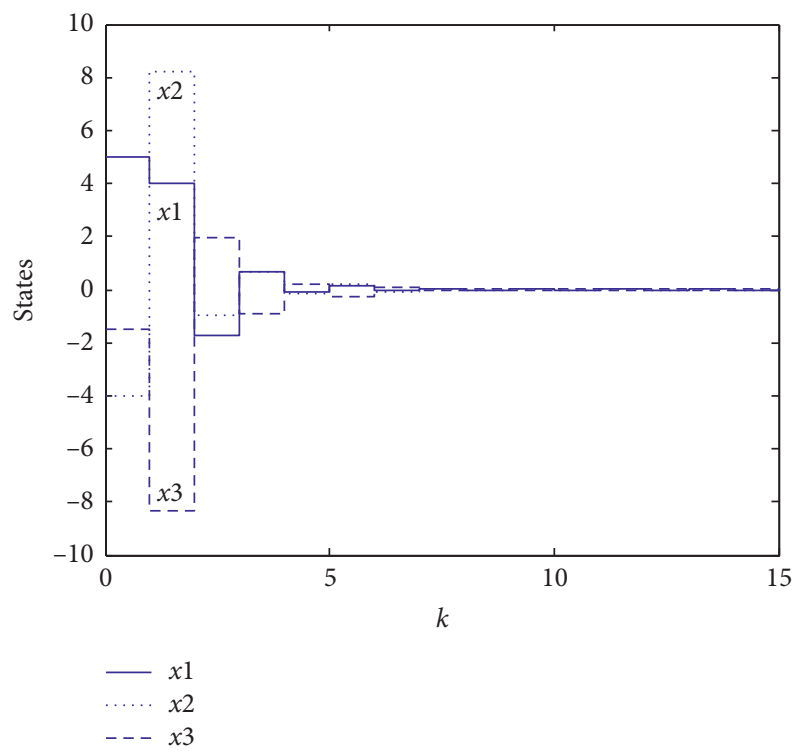

FIgURE 2: States' responses with the control input for Example 2.

feedback control with the obtained gain matrix is simulated again. Also, the states' trajectories and the control input are depicted in Figures 2 and 3, respectively. It is shown that all the controlled states $x_{i}(k), \forall i$, are surely convergent to zero in a short period.

\section{Conclusions}

In this work, we have investigated the admissibility analysis and the admissibilizing controller design for the uncertain discrete singular system with delayed state. First, based on Lyapunov-Krasovskii stability theory, an original admissibility condition for the nominal singular delay system was presented. In the sequel, we dealt with the admissibility

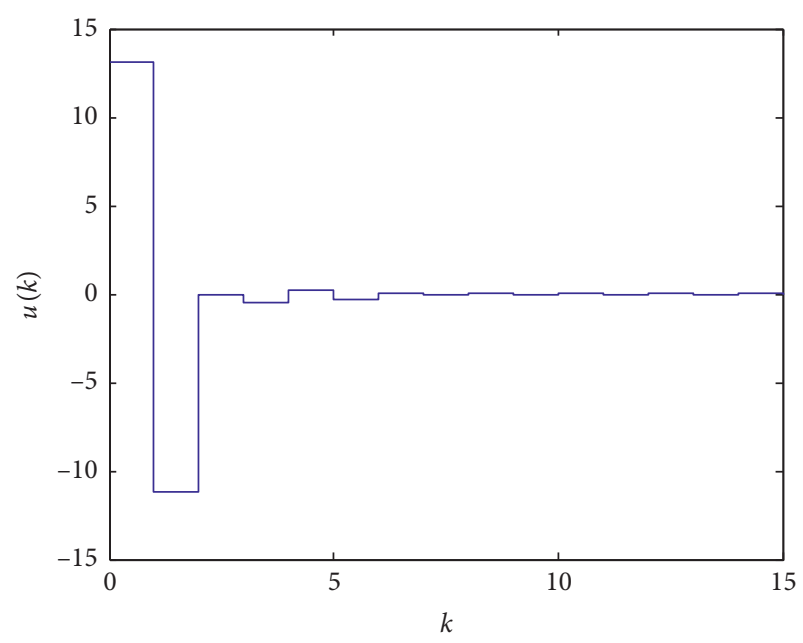

FIgURE 3: State feedback control input for Example 2.

analysis by hiring the LMI method. Furthermore, by involving the state feedback control law, we further discussed the admissibilizing controller design for the resulting closedloop system which can contain parametric uncertainties in the difference and systems' matrices simultaneously. Since all the presented criteria are represented in terms of strict LMIs or parametric LMIs, we can handily evaluate feasible solutions via the current LMI programming tools. Finally, the proposed result was demonstrated to be effective and practicable by the two examples given. However, the proposed analysis and controller design criteria are proposed for admissibility assurance of the considered systems. The future work will be suggested forward to involving some control objectives of specific admissibility assurance with the specific decay rate, damping, settle time, and so on.

\section{Data Availability}

No data were used to support this study.

\section{Conflicts of Interest}

The author declares that there are no conflicts of interest.

\section{References}

[1] G. Verghese, B. Levy, and T. Kailath, "A generalized statespace for singular systems," IEEE Transactions on Automatic Control, vol. 26, no. 4, pp. 811-831, 1981.

[2] L. Dai, "Singular control systems," Lecture Notes in Control and Information Sciences, Vol. 118, Springer-Verlag, New York, NY, USA, 1989.

[3] D. G. Luenberger and A. Arbel, "Singular dynamical Leotief systems," Econometrica, vol. 5, pp. 991-995, 1971.

[4] L. Jódar and P. Merello, "Positive solutions of discrete dynamic Leontief input-output model with possibly singular capital matrix," Mathematical and Computer Modelling, vol. 52, no. 7-8, pp. 1081-1087, 2010.

[5] R. Newcomb, "The semistate description of nonlinear timevariable circuits," IEEE Transactions on Circuits and Systems, vol. 28, no. 1, pp. 62-71, 1981. 
[6] D. Luenberger, "Dynamic equations in descriptor form," IEEE Transactions on Automatic Control, vol. 22, no. 3, pp. 312-321, 1977.

[7] F. L. Lewis, "A survey of linear singular systems," Circuits, Systems, and Signal Processing, vol. 5, no. 1, pp. 3-36, 1986.

[8] S. Xu and J. Lam, Robust Control and Filtering of Singular Systems, Springer, Berlin, Germany, 2006.

[9] I. Masubuchi, Y. Kamitane, A. Ohara, and N. Suda, " $H_{\infty}$ control for descriptor systems: a matrix inequalities approach," Automatica, vol. 33, no. 4, pp. 669-673, 1997.

[10] J.-H. Chou and W.-H. Liao, "Stability robustness of continuous-time perturbed descriptor systems," IEEE Transactions on Circuits and Systems I, vol. 46, no. 9, pp. 1153-1155, 1999.

[11] S. Xu and C. Yang, "Stabilization of discrete-time singular systems: a matrix inequalities approach," Automatica, vol. 35, no. 9, pp. 1613-1617, 1999.

[12] S. Xu and J. Lam, "Robust stability and stabilization of discrete singular systems: an equivalent characterization," IEEE Transactions on Automatic Control, vol. 49, no. 4, pp. 568574, 2004.

[13] I. Masubuchi, "Output feedback controller synthesis for descriptor systems satisfying closed-loop dissipativity," Automatica, vol. 43, no. 2, pp. 339-345, 2007.

[14] Y. T. Juang, C. P. Huang, and H. L. Lin, "Robust stability of discrete singular systems with delayed state," Journal of the Chinese Institute of Engineers, vol. 31, no. 1, pp. 149-155, 2008.

[15] G. Zhang, Y. Xia, and P. Shi, "New bounded real lemma for discrete-time singular systems," Automatica, vol. 44, no. 3, pp. 886-890, 2008.

[16] M. S. Mahmoud and H. E. Emara-Shabaik, "Reduced-order $H_{\infty}$ filter for singular discrete-time systems using strict LMIs," Mediterranean Journal of Measurement and Control, vol. 5, no. 4, pp. 155-163, 2009.

[17] S. Tian, Q. Liu, X. Dai, and J. Zhang, "A PD-type iterative learning control algorithm for singular discrete systems," Advances in Difference Equations, vol. 2016, Article ID 321, 9 pages, 2016.

[18] J. Wu, F. Liao, Y. Shao, S. Gao, H. Wang, and Z. Xu, "Robust preview control for uncertain discrete singular systems," Mathematical Problems in Engineering, vol. 2018, Article ID 1096793, 9 pages, 2018.

[19] G. Rajchakit, "Robust stability and stabilization of nonlinear uncertain stochastic switched discrete-time systems with interval time-varying delays," Applied Mathematics \& Information Sciences, vol. 6, no. 3, pp. 555-565, 2012.

[20] G. Rajchakit, "Switching design for the robust stability of nonlinear uncertain stochastic switched discrete-time systems with interval time-varying delay," Journal of Computational Analysis and Applications, vol. 16, no. 1, pp. 10-19, 2014.

[21] S. Mobayen and J. Ma, "Robust finite-time composite nonlinear feedback control for synchronization of uncertain chaotic systems with nonlinearity and time-delay," Chaos, Solitons \& Fractals, vol. 114, pp. 46-54, 2018.

[22] S. Rasoolinasab, S. Mobayen, A. Fekih, P. Narayan, and Y. Yao, "A composite feedback approach to stabilize nonholonomic systems with time varying time delays and nonlinear disturbances," ISA Transactions, vol. 101, pp. 177-188, 2020.

[23] C.-P. Huang, "Admissibility and design issues for discrete fuzzy singular delay systems with multiple difference matrices," International Journal of Innovative Computing, Information and Control, vol. 16, no. 5, pp. 1497-1510, 2020.

[24] S. Xu, J. Lam, and L. Zhang, "Robust $D$-stability analysis for uncertain discrete singular systems with state delay," IEEE
Transactions on Circuits and Systems I, vol. 49, no. 4, pp. 551-555, 2002.

[25] S.-H. Chen, "Robust D-stability analysis for linear discrete singular time-delay systems with structured and unstructured parameter uncertainties," IMA Journal of Mathematical Control and Information, vol. 20, no. 2, pp. 153-165, 2003.

[26] S.-H. Chen and J.-H. Chou, "Robust eigenvalue-clustering in a specified circular region for linear uncertain discrete singular systems with state delay," Applied Mathematics and Computation, vol. 186, no. 2, pp. 1660-1670, 2007.

[27] S. Ma, C. Cheng, and C. Zhang, "Delay-dependent robust stability and stabilisation for uncertain discrete singular systems with time-varying delays," IET Control Theory \& Applications, vol. 1, no. 4, pp. 1086-1095, 2007.

[28] S. Xu, J. Lam, Y. Zou, and J. Li, "Robust admissibility of timevarying singular systems with commensurate time delays," Automatica, vol. 45, no. 11, pp. 2714-2717, 2009.

[29] X. Sun, Q. Zhang, C. Yang, and Z. Su, "Stability analysis and stabilization for discrete-time singular delay systems," Journal of Systems Engineering and Electronics, vol. 22, no. 3, pp. 482-487, 2011.

[30] Z. Feng, W. Li, and J. Lam, "New admissibility analysis for discrete singular systems with time-varying delay," Applied Mathematics and Computation, vol. 265, pp. 1058-1066, 2015.

[31] Y. Cui, J. Lam, Z. Feng, and J. Shen, "Robust admissibility and admissibilisation of uncertain discrete singular time-delay systems," International Journal of Systems Science, vol. 47, no. 15, pp. 3720-3729, 2016.

[32] N. H. Muoi, G. Rajchakit, and V. N. Phat, "LMI approach to finite-time stability and stabilization of singular linear discrete delay systems," Acta Applicandae Mathematicae, vol. 146, no. 1, pp. 81-93, 2016.

[33] P. Gu, S. Tian, and Q. Liu, "Iterative learning control for discrete singular time-delay systems," IMA Journal of Mathematical Control and Information, vol. 36, no. 02, pp. 623-637, 2019.

[34] R. Hajmohammadi and S. Mobayen, "An efficient observer design method for singular discrete-time systems with time delays and nonlinearity: LMI approach," Scientia Iranica D, vol. 26, no. 3, pp. 1690-1699, 2019.

[35] D. Wang, S. Han, and J. Chen, "Robust admissibilization for discrete-time singular systems with time-varying delay," Mathematical Problems in Engineering, vol. 2019, Article ID 5368013, 14 pages, 2019.

[36] S. Mobayen, F. Bayat, H. Omidvar, and A. Fekih, "Robust global controller design for discrete-time descriptor systems with multiple time-varying delays," International Journal of Robust and Nonlinear Control, vol. 30, no. 7, pp. 2809-2831, 2020.

[37] C. Lin, J. L. Wang, G.-H. Yang, and J. Lam, "Robust stabilization via state feedback for descriptor systems with uncertainties in the derivative matrix," International Journal of Control, vol. 73, no. 5, pp. 407-415, 2000.

[38] C. Lin, J. Lam, J. Wang, and G.-H. Yang, "Analysis on robust stability for interval descriptor systems," Systems \& Control Letters, vol. 42, no. 4, pp. 267-278, 2001.

[39] C. Lin, Q.-G. Wang, and T. H. Lee, "Robust normalization and stabilization of uncertain descriptor systems with normbounded," IEEE Transactions on Automatic Control, vol. 50, no. 7, pp. 515-520, 2005.

[40] C.-P. Huang, "Specific D-admissibility and design issues for uncertain descriptor systems with parametric uncertainty in the derivative matrix," Mathematical Problems in Engineering, vol. 2016, Article ID 6142848, 12 pages, 2016. 
[41] S. Boyd, L. El Ghaoui, E. Feron, and V. Balakrishnan, Linear Matrix Inequalities in Systems and Control Theory, SIAM, Philadelphia, PA, USA, 1994.

[42] P. Gahinet, A. Nemirovski, A. J. Jaub, and M. Chilali, LMI Control Toolbox User's Guide, The Mathworks Partner Series, Natick, MA, USA, 1995. 